\title{
Control of structure, size and density of Ge dot on Si (1 00$)$ through multistep procedure
}

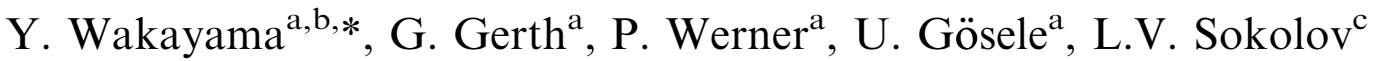 \\ ${ }^{a}$ Max-Planck Institute of Microstructure Physics, Weinberg 2, D-06120 Halle, Germany \\ ${ }^{\mathrm{b}}$ National Institute for Materials Science, Nanomaterials Laboratory, 2268-1 Shimo-shidami, Moriyama-ku, Nagoya 463-0003, Japan \\ ${ }^{\mathrm{c}}$ Institute of Semiconductor Physics, Russian Academy of Science, Siberian Branch, Lavrentieva 13, 630090 Novosibirsk, Russia
}

Received 13 April 2001; accepted 11 June 2001

Communicated by $\mathrm{T}$. Nishinaga

\begin{abstract}
We propose a multistep procedure for Ge dot growth on Si (100) substrates. This procedure includes (i) submonolayer $\mathrm{C}$ deposition on a Ge wetting layer or a Ge surface, (ii) Ge deposition at low temperature and (iii) postannealing after $\mathrm{Ge}$ and $\mathrm{C}$ deposition for controlling size, structure and density of the Ge dots. The main effect of $\mathrm{C}$ on Ge wetting layer was to enhance a structure transition of the Ge dot. Meanwhile, C on the Ge surface was found to suppress a structure modification of the Ge dots against post-annealing. The structure of Ge layers deposited at low temperature was readily modified upon post-annealing. By combining these procedures and optimizing experimental conditions, $10 \mathrm{~nm}$-sized Ge dots with a high number density in the order of $10^{11} \mathrm{~cm}^{-2}$ were successfully grown on the Si (1 00 ) substrate. (C) 2001 Elsevier Science B.V. All rights reserved.
\end{abstract}

PACS: 68.35.-p; 81.15.-z; 81.05.Cy

Keywords: A1. Nanostructures; A3. Molecular beam epitaxy; B2. Semiconducting silicon; B2. Semiconducting germanium

\section{Introduction}

Nanometer-scaled materials have attracted much attention because of their outstanding properties based on quantum-size effects. A selfassembly process has been applied to fabricate such nanoscaled materials. The initial stage of thin film growth is utilized to form a three-dimensional

\footnotetext{
*Corresponding author. Tel.: +81-52-736-6011; fax: + 8152-736-6012.

E-mail address: WAKAYAMA.yutaka@nims.go.jp (Y. Wakayama).
}

(3D) structure for quantum dots, which is wellknown as Stranski-Krastanov (SK) growth mode. Then, the elastic energy caused by the lattice mismatch between the substrates and the films is a driving force for the 3D growth. In particular, the $\mathrm{Si}-\mathrm{Ge}$ system, which includes $4 \%$ lattice mismatch, has been intensively studied from the viewpoint of both fundamental and applied science [1-3]. The Ge growth on the Si substrate shows typical SK mode, i.e., the 3D dot growth subsequent to a $2 \mathrm{D}$ wetting layer growth. However, the $\mathrm{Ge}$ dot on $\mathrm{Si}$ shows a quite complex aspect. Various structures, which are so-called hut, 
pyramid and dome structures, are grown at the same time [4,5]. Understanding this complicated growth mechanism is one of the most important issues [6,7], whereas the details are still unclear at the moment.

Each Ge dot structure has both advantages and disadvantages with respect to the quantum dots. For example, the hut structure has a few nanometer heights, which is available to achieve a carrier confinement effect. However, the lateral size is widely distributed and uncontrollable. On the contrary, the dome structure shows quite high size uniformity, but its size is too large for the quantum dots. The size is more than $100 \mathrm{~nm}$ in diameter [8]. The pyramid structure is formed at somewhat higher temperature than that of the hut structure. The temperature range for the pyramid growth is limited. As a result, the pyramid structure coexists with other structures. Therefore, to establish a new technique to control the Ge dot structures, including shape, density, size and size uniformity as well, is an indispensable task to attain the quantum dot formation in the $\mathrm{Ge}-\mathrm{Si}$ system.

Recently, Schmidt and co-workers [9-11] dealt with this difficult problem. Very small Ge domes with 10-20 nm in diameter was successfully formed by depositing a sub-monolayer $\mathrm{C}$ on the $\mathrm{Si}$ substrate. They have intensively investigated photoluminescence properties of thus prepared $\mathrm{C}$-induced $\mathrm{Ge}$ dots. After their report, $\mathrm{C}$ has been recognized as an essential material for controlling Ge dot structure on nanometer scale $[12,13]$. However, further investigation is still required for detailed understanding of the $\mathrm{C}$ effect to develop the Ge dot growth technique.

On the basis of these backgrounds, we have investigated new methods for controlling the $\mathrm{Ge}$ dot growth. The main purpose of this article is to discuss the growth processes, in particular Crelated growth processes, of Ge dots in detail. Additionally, we inquired into new techniques to design $10 \mathrm{~nm}$-sized $\mathrm{Ge}$ dots with high number density. For this purpose, we took multistep procedures: sub-monolayer $\mathrm{C}$ deposition (i) on the Ge wetting layer, (ii) on the Ge surface, (iii) Ge deposition at low temperature and (iv) postannealing after $\mathrm{Ge}$ and $\mathrm{C}$ deposition.
First of all, we examined the effect of each procedure. The pure Ge layers deposited on the Si substrates were annealed to study the postannealing effect on the Ge dot structure, which are denoted as samples-I. The Ge layers, in particular deposited at low temperature, showed drastic structure change upon post-annealing. Next, in order to obtain more insight of the $\mathrm{C}$ influence on the Ge dot structure, sub-monolayers $\mathrm{C}$ were deposited on the Ge wetting layers or on the Ge surface, those are denoted as samples-II and III, respectively. As a result, The submonolayers $\mathrm{C}$ were found to have distinct effects on the Ge dot structure. The $\mathrm{C}$ layer on the $\mathrm{Ge}$ wetting layer enhanced dome structure formation instead of the hut structure even at relatively low substrate temperature. On the other hand, the C layer on the Ge surface suppressed the structure modification upon post-annealing. Based on these experimental results, we examined combinations of each procedure that provided us with various recipes to prepare the Ge dots on the Si substrate.

\section{Experimental conditions}

Boron doped p-type Si (1 00$)$ wafers were used for the substrates. After chemical cleaning, the Si (100) substrates were introduced into an ultra high vacuum (UHV) chamber with a background pressure of $1 \times 10^{-10}$ Torr. Then, the temperature of the substrates increased up to $830^{\circ} \mathrm{C}$ and was kept at that temperature for $20 \mathrm{~min}$ to remove oxide layers. A well-defined $(2 \times 1)$ pattern of reflection high-energy electron diffraction (RHEED) was observed after heat-treatment, which indicated a flat and clean surface of the Si substrate.

$\mathrm{Si}$ and Ge were deposited by a solid source molecular beam epitaxy machine (Riber Siva 45) equipped with electron-beam evaporators. The deposition of $\mathrm{C}$ was carried out through sublimation from a heated $\mathrm{C}$ filament. Typical deposition rates of $\mathrm{Si}, \mathrm{Ge}$ and $\mathrm{C}$ were $0.1,0.05$ and $6.4 \times 10^{-5} \AA / \mathrm{s}$, respectively. The Si buffer layer with $10 \mathrm{~nm}$ in thickness was deposited at $450^{\circ} \mathrm{C}$.

We prepared three different groups of samples, which are denoted as samples-I, II and III. 
Samples-I were pure Ge layers with $6 \AA$ nominal thickness deposited on $\mathrm{Si}(100)$. The C layer was not deposited for these specimens. Thermal annealing was carried out in UHV immediately after Ge deposition to examine the post-annealing effect.

For samples-II, the Ge layers with $4 \AA$ thickness were deposited on $\mathrm{Si}$ for the wetting layers at the beginning. Subsequently, the $\mathrm{C}$ layers, of which thickness was ranging from 0.01 monolayer (ML) to $0.10 \mathrm{ML}$, were deposited on the wetting layer. Finally, Ge top layers were deposited. The thickness of the Ge top layer was $2 \AA$, that is, the total thickness of Ge was fixed to $6 \AA$.

For samples-III, the Ge layers with $6 \AA$ in thickness were deposited on Si (1 00$)$ first of all. Then, the sub-monolayers $\mathrm{C}$ were deposited on top of the Ge surface. The thickness of the $\mathrm{C}$ top layers was ranging from 0.05 to $0.15 \mathrm{ML}$. The postannealing was carried out for all samples-III immediately after $\mathrm{C}$ deposition in the UHV.

Morphology of the samples was monitored by RHEED during the deposition. Structures of the samples thus prepared were examined by tappingmode atomic force microscopy (AFM) and highresolution transmission electron microscopy (TEM).

\section{Results and discussion}

\subsection{Structure transition upon post-annealing}

First of all, pure Ge layers with $6 \AA$ thickness were deposited and were post-annealed in order to examine the post-annealing effect on the $\mathrm{Ge}$ dot structure. Fig. 1 shows AFM images of the Ge dots on the $\mathrm{Si}$ substrates deposited at (a) $500^{\circ} \mathrm{C}$ and followed by post-annealing at (b) $540^{\circ} \mathrm{C}$, (c) $550^{\circ} \mathrm{C}$ and (c) $560^{\circ} \mathrm{C}$, respectively. The temperature of the substrates was raised up to each postannealing temperature immediately after deposition and was kept at those temperatures for $5 \mathrm{~min}$. Then, the samples were cooled down. All AFM images were obtained at room temperature.

In Fig. 1(a), the as-deposited Ge dots show the typical hut structure; rectangular with edges along the $\left[\begin{array}{lll}0 & 0 & 1\end{array}\right]$ orientation. The hut structure was stable upon post-annealing up to $540^{\circ} \mathrm{C}$ for $5 \mathrm{~min}$ and was maintained their shape as can be seen in Fig. 1(b). However, the Ge dot structure was abruptly changed to domes with round shape and pyramids with square bases by post-annealing above $550^{\circ} \mathrm{C}$. This structure transition can be seen in Figs. 1(c) and (d). Namely, the Ge dots structure was drastically changed through postannealing within a quite narrow temperature range of $540-560^{\circ} \mathrm{C}$.

The size of domes and pyramids is strongly dependent on the post-annealing temperature. In a broad way, the $\mathrm{Ge}$ dots of $560^{\circ} \mathrm{C}$ have $20 \%$ larger diameter of domes and 50\% larger square base of pyramids than those of $550^{\circ} \mathrm{C}$. On the other hand, the number density of the Ge dots decreases with increasing post-annealing temperature. Particularly, few Ge pyramids were observed around the large Ge domes as indicated by a circle in Fig. 1(d). That is, the structure modification occurred through Ostwald ripening during post-annealing.

These results suggest that control of the Ge dot structures, including their size, shape and number density, is quite difficult only by post-annealing. Similar difficulty in controlling Ge dot structures was observed when the substrate temperature is changed during the Ge deposition [5,14].

Next, we deposited Ge layers at relatively low temperature and inquired into their structure stability upon thermal annealing. Fig. 2 shows AFM images of the Ge dots on the Si substrate deposited at $350^{\circ} \mathrm{C}$. An as-deposited Ge is shown in Fig. 2(a). Here, a slight undulation with $50 \mathrm{~nm}$ in diameter and $1-2 \mathrm{~nm}$ in height was observed. In general, the SK growth of the Ge dots can be observed at the temperature range of around 450 $650^{\circ} \mathrm{C}$. Meanwhile, the structure of the Ge layer on Si gradually transits from $3 \mathrm{D}$ to $2 \mathrm{D}$ as substrate temperature decreases [15-17]. Such structure is formed through the step-flow mechanism at low temperature range [18]. Roughly speaking, the structure of $350^{\circ} \mathrm{C}$ sample can be regarded as twodimensional from the viewpoint of their high aspect ratio.

Fig. 2(b) shows Ge post-annealed at $390^{\circ} \mathrm{C}$ subsequent to $350^{\circ} \mathrm{C}$ deposition. Post-annealing even at $390^{\circ} \mathrm{C}$ caused extreme structural change as can be seen in Fig. 2(b). Very large Ge islands 

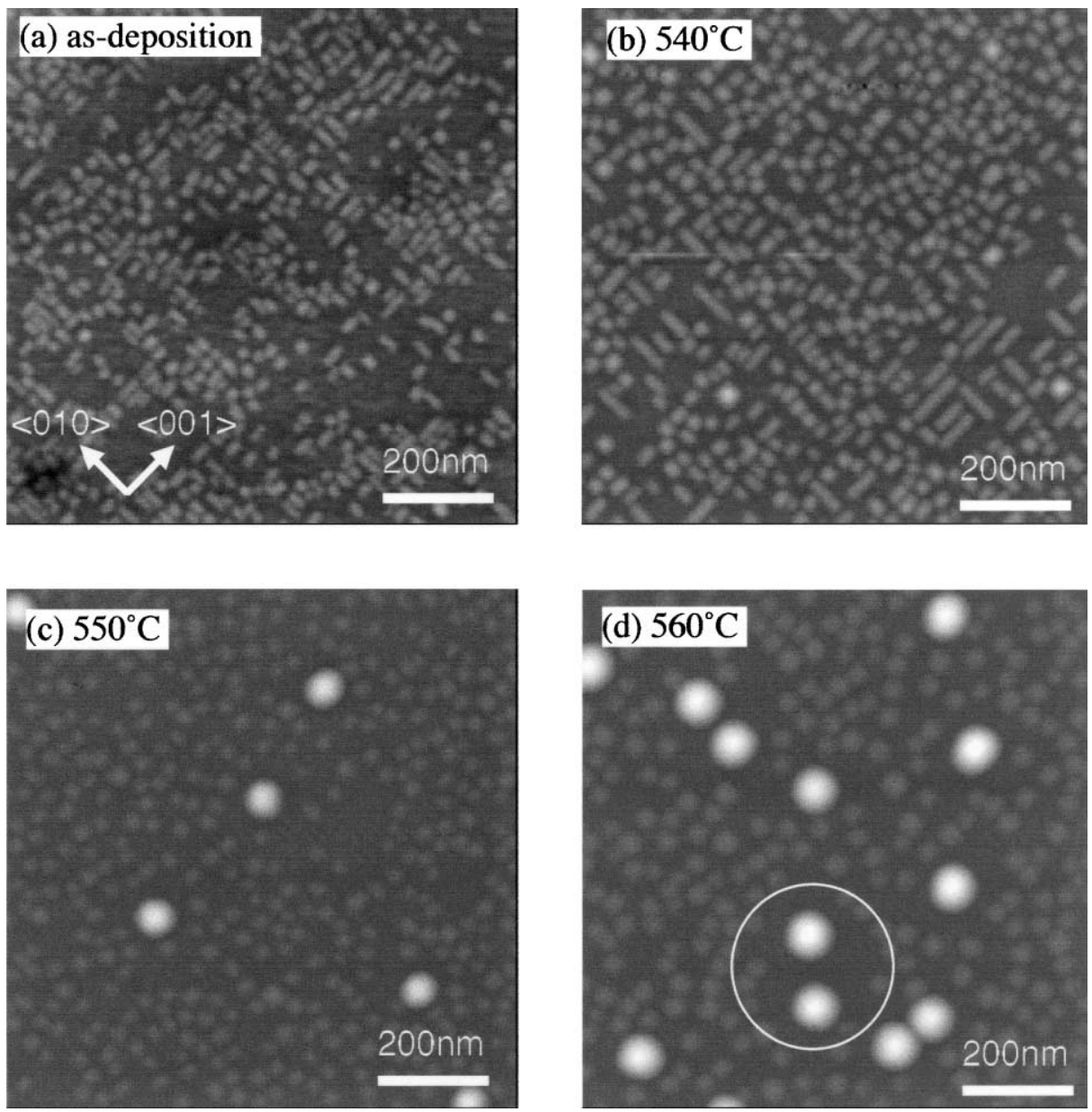

Fig. 1. AFM images of $6 \AA$ Ge deposited at $500^{\circ} \mathrm{C}$ on $\mathrm{Si}(100)$ : (a) as-deposited Ge shows a hut structure. The temperatures of postannealing are (b) $540^{\circ} \mathrm{C}$, (c) $550^{\circ} \mathrm{C}$ and (d) $560^{\circ} \mathrm{C}$. The annealing condition of $540^{\circ} \mathrm{C}$ for 5 min was insufficient to give rise to the structure transition, meanwhile the Ge dot structure started to transform to domes and pyramids above $550^{\circ} \mathrm{C}$. At higher temperature, few dots were observed around domes as indicated by a circle in Fig. 1(d), suggesting that the structure transition occurred through Ostwald ripening.

without any facets and orientations were formed. These are considered to be formed through agglomeration of surface diffusing Ge atoms during post-annealing. This result indicates that the $\mathrm{Ge}$ deposited at $350^{\circ} \mathrm{C}$ is quite unstable upon thermal annealing compared with that deposited at $500^{\circ} \mathrm{C}$.

As mentioned above, the driving force of $3 \mathrm{D}$ growth in SK mode is an elastic energy caused by the lattice mismatch between substrates and deposited materials. The lattice of the $3 \mathrm{D}$ Ge dots is persistently coherent to that of the Si substrate to minimize the total energy at the expense of the surface energy. In this case, the $3 \mathrm{D}$ dots as well as the $2 \mathrm{D}$ wetting layers grow without any defects. We consider that such a highly strained but defectfree structure is the reason for the Ge huts grown at $500^{\circ} \mathrm{C}$ to be relatively stable against postannealing up to $540^{\circ} \mathrm{C}$.

On the other hand, the lattice of the Ge films formed at low temperature is elastically relaxed by introducing some defects, such as point defects in 

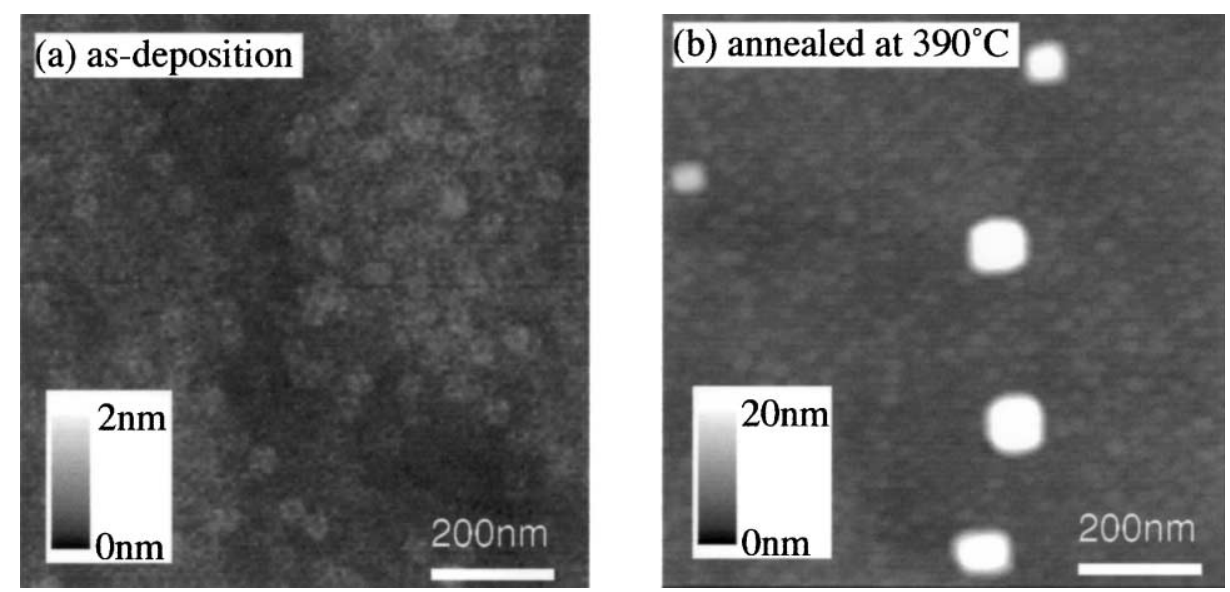

Fig. 2. AFM images of $6 \AA$ Ge deposited at $350^{\circ} \mathrm{C}$ on $\mathrm{Si}(100)$ : (a) as-deposited Ge shows a slight undulation, which is a "quasi-2D structure"; (b) post-annealed $\mathrm{Ge}$ at $390^{\circ} \mathrm{C}$ form large $3 \mathrm{D}$ islands, suggesting that the Ge layer deposited at low temperature is unstable against post-annealing.

the Ge layer [19]. We suppose that such relaxed structure with the defects is responsible to the instability of the Ge layer and allows the Ge layers to reconstruct drastically upon post-annealing even at $390^{\circ} \mathrm{C}$.

\subsection{Sub-monolayer $C$ on Ge wetting layer}

As described in the previous section, the Ge dot structure control on nanometer scale cannot be achieved only through post-annealing. In order to overcome this difficulties, the sub-monolayer $\mathrm{C}$ were deposited on the Ge wetting layer.

Fig. 3 shows AFM images of the Ge dots with the $\mathrm{C}$ layers on the wetting layers. Both $\mathrm{Ge}$ and $\mathrm{C}$ were deposited at $500^{\circ} \mathrm{C}$. The mean thickness of $\mathrm{C}$ layers were (a) $0.01 \mathrm{ML}$, (b) $0.03 \mathrm{ML}$ and (c) $0.05 \mathrm{ML}$, respectively. The setup of the samples-II was schematically illustrated in this figure. The Ge dots with 0.01 ML C on the wetting layer still indicate the hut structure as shown in Fig. 3(a), whereas the average length of each hut became short. Meanwhile, the structure of the Ge dots gradually transformed from huts to domes with increasing thickness of the $C$ layer as can be seen in Figs. 3(b) and (c). Note that, the size of the domes is quite uniform, $40 \mathrm{~nm}$ in diameter and $5 \mathrm{~nm}$ in height on average, and is independent of the thickness of $\mathrm{C}$ layer. Therefore, the essential effect of $\mathrm{C}$ on the wetting layer is to enhance the structure transition of the Ge dots from hut to dome.

Fig. 4 shows AFM images of other types of samples-II, in which the Ge top layers were deposited at different temperature. The substrate temperature for the wetting layers and the $\mathrm{C}$ layers deposition was fixed at $500^{\circ} \mathrm{C}$. The Ge top layers with $2 \AA$ nominal thickness were deposited at (a) $400^{\circ} \mathrm{C}$, (b) $450^{\circ} \mathrm{C}$ and (c) $500^{\circ} \mathrm{C}$, respectively. The $\mathrm{C}$ layer thickness was $0.10 \mathrm{ML}$ for all samples.

The dome structure was observed even below $450^{\circ} \mathrm{C}$ due to the $\mathrm{C}$ layer effect as shown in Figs. 4(a) and (b), although only hut structure was formed below $500^{\circ} \mathrm{C}$ for the pure Ge. A TEM image of a typical dome structure is shown inset. The diameter of which is about $15 \mathrm{~nm}$. The size of the Ge dot decreased and the number density increased as the temperature for the Ge top layer decreased. As mentioned above, the $\mathrm{C}$ layer has influence on the Ge dot structure but not on the dot size. On the other hand, the size and density of the Ge dot is mainly determined by the substrate temperature for the top layer deposition when the thickness of the $\mathrm{C}$ layer is kept constant.

For these samples, the wetting layers deposited at $500^{\circ} \mathrm{C}$ provide a strain field to initiate subsequent $3 \mathrm{D}$ growth. Then, the $\mathrm{C}$ layers enhance the dome formation. The low temperature for the top 

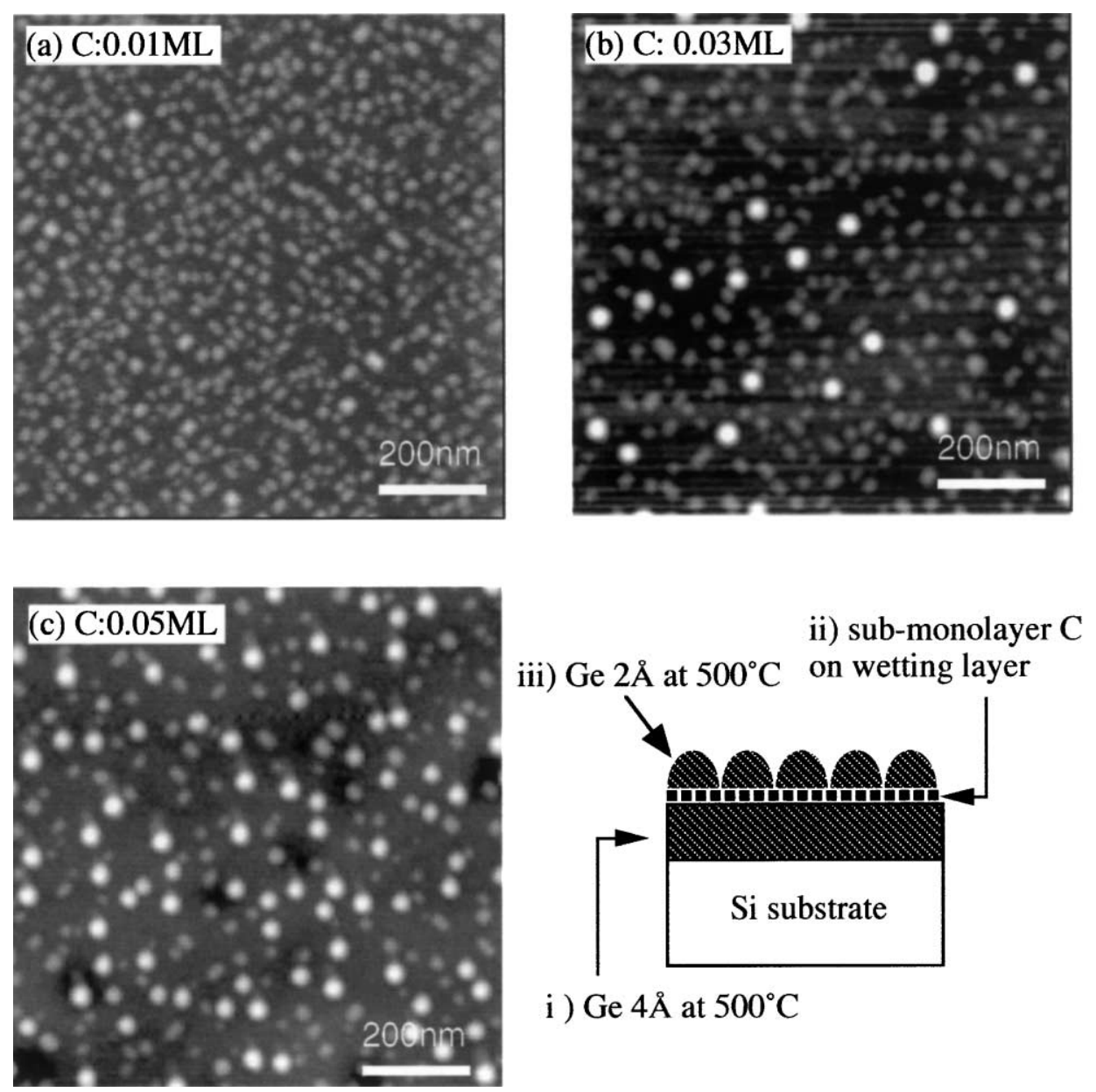

Fig. 3. AFM images of Ge dots with sub-monolayer $\mathrm{C}$ on the Ge wetting layers. All samples were prepared at $500^{\circ} \mathrm{C}$. The mean thickness of the C layers are (a) $0.01 \mathrm{ML}$, (b) $0.03 \mathrm{ML}$ and (c) $0.05 \mathrm{ML}$. The Ge dot structure transited from huts to domes with increasing thickness of the $\mathrm{C}$ layer. An illustration shows a setup of the samples.

layer deposition leads to the dome size reduction and to the increase in the number density. In Fig. 4(a), the size and density of Ge dot were 5$20 \mathrm{~nm}$ and $1 \times 10^{11} \mathrm{~cm}^{-2}$, respectively. Consequently, the three-step procedure: the Ge wetting layer at $500^{\circ} \mathrm{C}$, the sub-monolayer $\mathrm{C}$ at $500^{\circ} \mathrm{C}$ and the Ge top layer at low temperature, is an effective technique to form small Ge dot with high density. However, the size uniformity is not satisfactory. In Fig. 4(b), some dots show round shape with homogeneous size, which is dome structure. Meanwhile, many other dots, which have relatively small size, show irregular shape. For these dots, no specific facets were observed. The detail concerning $\mathrm{Ge}$ dot structure for these samples were discussed elsewhere [20].

For further improvement of the Ge dot size and their uniformity, we examined a four-step procedure: (i) $\mathrm{Ge}$ wetting layer at $500^{\circ} \mathrm{C}$, (ii) submonolayer $\mathrm{C}$ at $500^{\circ} \mathrm{C}$, (iii) Ge top layer at $350^{\circ} \mathrm{C}$ and (iv) post-annealing at $390^{\circ} \mathrm{C}$. Here, we applied the structure instability of the low-temperature deposited Ge layers. Fig. 5 shows AFM images of samples-II prepared by four-step procedure, of 

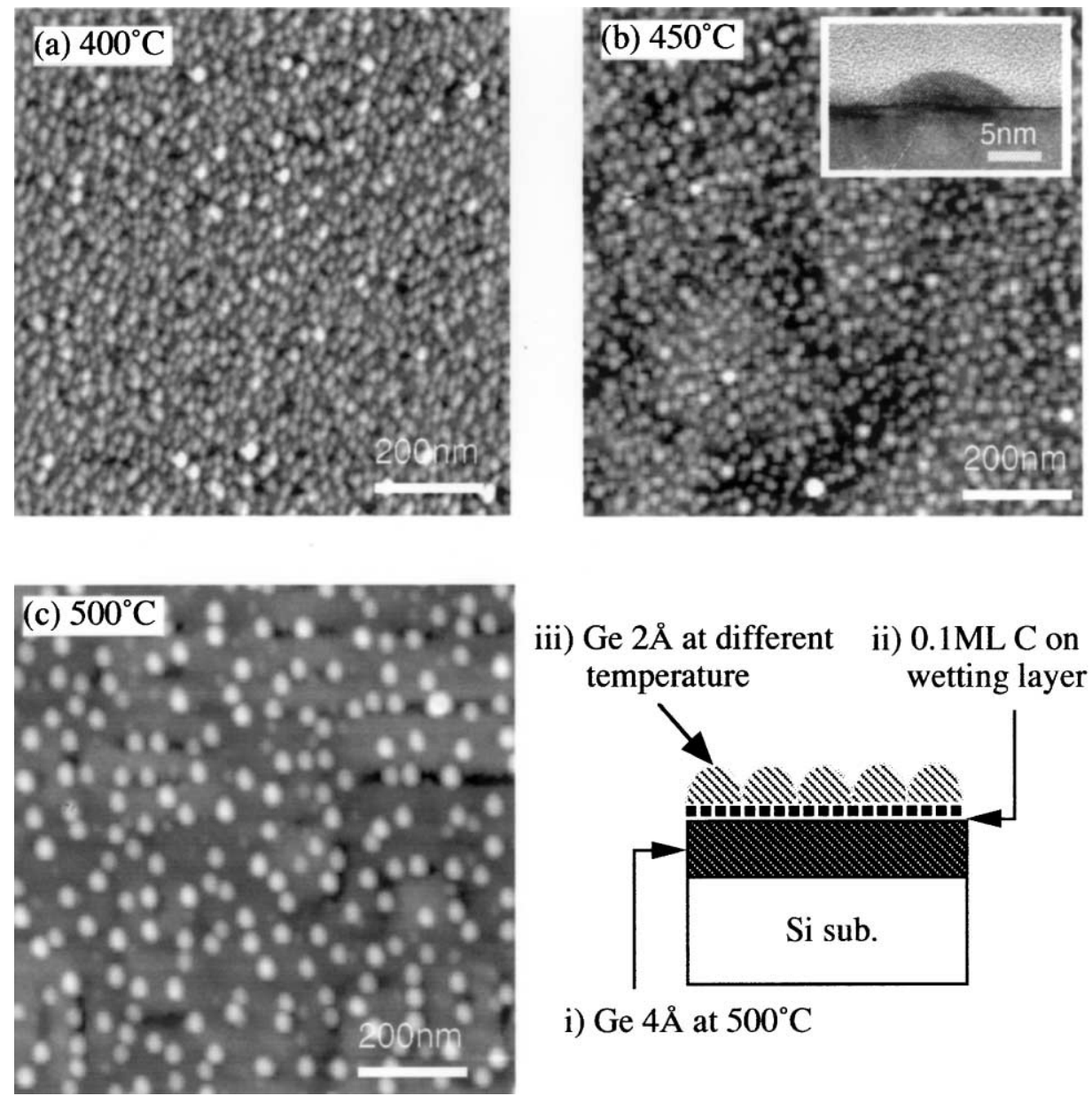

i) $\mathrm{Ge} 4 \AA$ at $500^{\circ} \mathrm{C}$

Fig. 4. AFM images of Ge dots. The temperature of the Ge top layer deposition were different. The Ge top layers were deposited at (a) $400^{\circ} \mathrm{C}$, (b) $450^{\circ} \mathrm{C}$ and (c) $500^{\circ} \mathrm{C}$. The Ge wetting layer with $4 \AA$ thickness and the sub-monolayer $\mathrm{C}$ with $0.1 \mathrm{ML}$ thickness were deposited at $500^{\circ} \mathrm{C}$. A TEM image of a typical dome structure with $15 \mathrm{~nm}$ in diameter is shown in Fig. 4(b). The sample setup is also illustrated.

which $\mathrm{C}$ thicknesses were (b) $0.05 \mathrm{ML}$ and (c) $0.10 \mathrm{ML}$, respectively. A sample without C layer was shown in Fig. 5(a) for comparison.

For the sample without $\mathrm{C}$, the surface was flat and no distinct 3D structure was observed as shown in Fig. 5(a). Three-dimensional islanding gradually started with increasing thickness of the $\mathrm{C}$ layers as can be seen in Figs. 5(b) and (c). In particular, Ge dots with $10-20 \mathrm{~nm}$ in size were homogeneously distributed on the $\mathrm{Si}$ substrate in the case of $0.10 \mathrm{ML} \mathrm{C}$ as shown in Fig. 5(c).
Whereas, the $\mathrm{C}$ layer with $0.05 \mathrm{ML}$ in thickness was insufficient for uniform distribution of $\mathrm{Ge}$ dots.

Here, the Ge wetting layer deposited at $500^{\circ} \mathrm{C}$ is stable against post-annealing at $390^{\circ} \mathrm{C}$ as demonstrated in Fig. 1. It contains a lattice strain, which trigger 3D growth for the Ge top layer. However, the Ge top layers don't form the 3D dots because of the low deposition temperature. The postannealing has the effect on reconstruction for such low temperature deposited layers. Therefore, only 

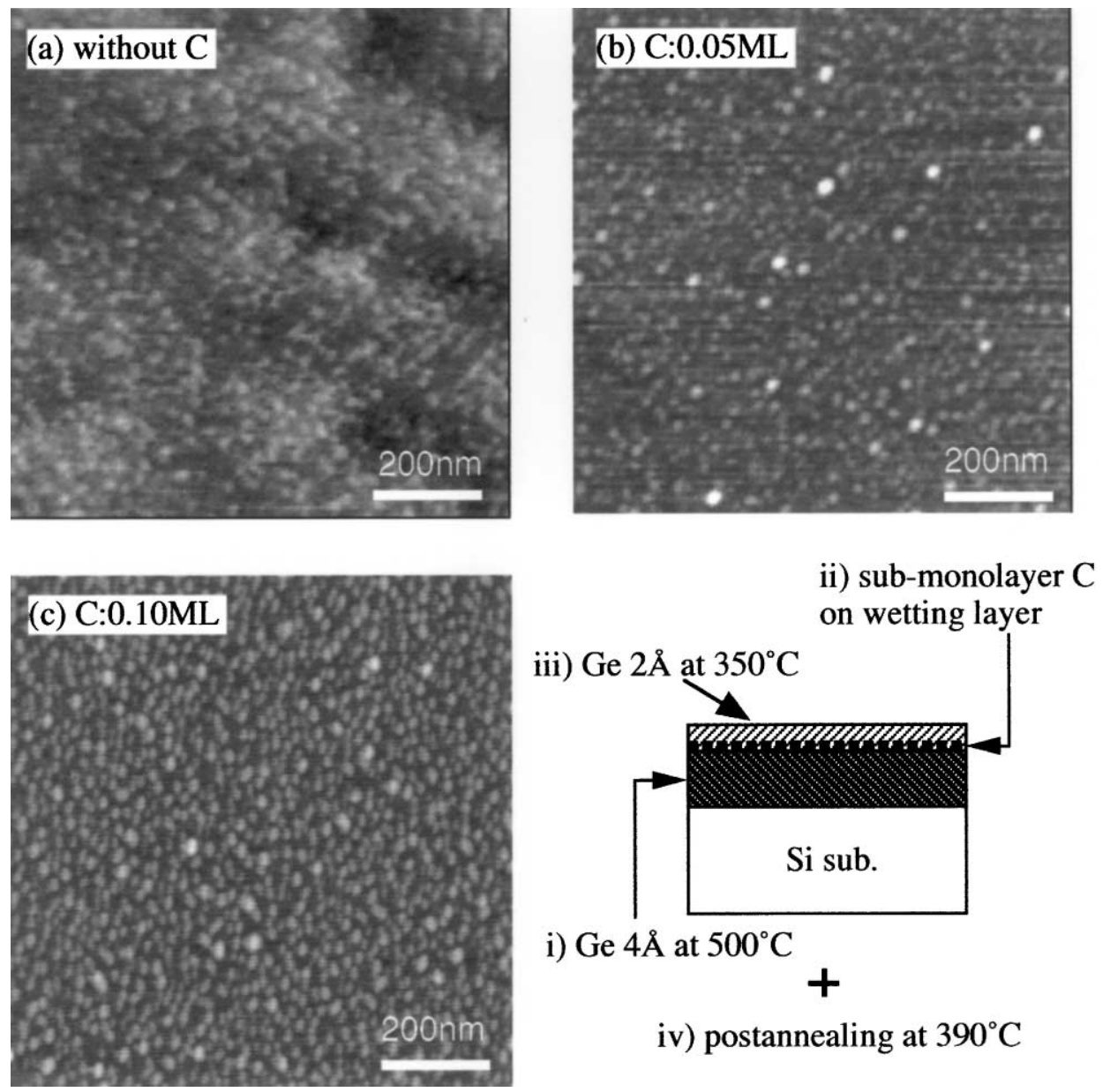

Fig. 5. AFM images of Ge dots with and without $\mathrm{C}$ layer on the Ge wetting layer. The deposition temperature for the Ge wetting layers and sub-monolayer $\mathrm{C}$ were $500^{\circ} \mathrm{C}$. The Ge top layers were deposited at $350^{\circ} \mathrm{C}$ and followed by post-annealing at $390^{\circ} \mathrm{C}$. (a) Without $\mathrm{C}$ layer, the surface remained flat even after post-annealing. (b) $0.05 \mathrm{ML} \mathrm{C}$ enhanced 3D islanding, although the dot distribution was inhomogeneous. (c) $0.10 \mathrm{ML} \mathrm{C}$ resulted in an uniform dot distribution.

$2 \AA \mathrm{Ge}$ of the top layers contributed to form 3D dots in these samples and, as a result, the large island observed in Fig. 2(b) was not formed. Then, the strain field in the wetting layer and the $C$ layers on the wetting layers as well promoted to $3 \mathrm{D}$ islanding.

Now, the question is why such a small amount of $\mathrm{C}$ can affect on the Ge dots structure modification. In order to explain the role of the $\mathrm{C}$ layer, it is essential to understand the reason for coexistence of some distinct Ge dots, i.e., hut, pyramid and dome. Medeiros-Ribeiro et al. [6] discussed the structure transition of the $\mathrm{Ge}$ dots by taking account of some parameters concerning configuration energies, such as a bulk strain, facet and interface energies and interaction among the facet edges. And they explained that both hut and dome are metastable structures and those configuration energies have minimum values. A certain activation energy is required to bring about the structure transition. In their article, medium sizes Ge dots that indicated multi-faceted surface were observed. 
They attributed these dots to the intermediate structure between hut and dome which were formed only on the way of structure transition.

We suppose that the $\mathrm{C}$ layers affect on the configuration energies, which determine the $\mathrm{Ge}$ dots structures. Particularly, the $\mathrm{C}$ layer is considered to influence on the interface energy between the wetting layer and the top layer of Ge. These are different materials with respect to strain energy because an extent of strains and their distribution is different. The $\mathrm{C}$ atoms at the wetting layer/dot interface form the $\mathrm{Ge}-\mathrm{C}$ bond and induce the lattice strain. Then, the $\mathrm{C}$ atoms may change the stability of the hut structure and the energy barrier between hut and dome, resulting in the enhancement of dome formation even at low temperature.

The C-induced Ge dots have been reported by Schmidt et al. [9-11] and Leifeld et al. [11,12]. In their investigations, the sub-monolayer $\mathrm{C}$ was deposited directly on the $\mathrm{Si}$ surface. Then, C atoms readily diffused into $\mathrm{Si}$ substrate to form a $\mathrm{Si}-\mathrm{C}$ alloy layer and to make the surface rough. The surface roughness, as a result, resulted in a reduction of the surface diffusibility of the $\mathrm{Ge}$ adatoms and in an increase in the nucleation probability. For this reason, growth of the Ge dots with small size and high density was achieved. On the contrary, the alloying between $\mathrm{C}$ atoms and $\mathrm{Ge}$ is negligible in our experiments because the amount of deposited $\mathrm{C}$ is quite smaller than those in Ref. [9-12]. Actually, a clear streak pattern of RHEED was observed even after $\mathrm{C}$ layer deposition on the Ge wetting layer as an evidence of the flat surface. Consequently, the surface roughness has no influence on the diffusibility of Ge adatoms, although the $\mathrm{C}$ layer may contribute to alter the surface energy of the Ge wetting layer [21].

\subsection{Sub-monolayer $C$ on Ge surface}

We examined the effect of sub-monolayer C, which were deposited on the Ge surface. Fig. 6 shows the AFM images of samples-III. The samples were prepared through a three-step procedure: (i) $6 \AA$ Ge layer at $500^{\circ} \mathrm{C}$, (ii) $\mathrm{C}$ top layers with different thickness at $500^{\circ} \mathrm{C}$ and (iii) post-annealing at $550^{\circ} \mathrm{C}$. The nominal thickness of the $\mathrm{C}$ top layer were (a) $0.15 \mathrm{ML}$, (b) $0.10 \mathrm{ML}$ and (c) $0.05 \mathrm{ML}$, respectively.

In Fig. 6(a), log narrow Ge islands are observed. The edges are along the $\left[\begin{array}{lll}0 & 0 & 1\end{array}\right]$ direction. Thus, these are regarded as the hut structure, while the Ge islands show no clear rectangular shape that were observed in the as-deposited hut structure shown in Fig. 1(a). As shown in Fig. 1(c), the all Ge huts were transited to domes and pyramids after post-annealing at $550^{\circ} \mathrm{C}$ for the pure Ge. On the contrary, the hut structure is dominant in Fig. 6(a) even after the same post-annealing. Namely, the structure transition from hut to dome was suppressed by the $\mathrm{C}$ top layer on the $\mathrm{Ge}$ surface.

The structure of the Ge dots gradually changed to round shape with decreasing thickness of the $\mathrm{C}$ top layer as can be seen in Figs. 6(b) and (c). Furthermore, a careful observation in an enlarged AFM image of $0.10 \mathrm{ML}$ sample, shown in Fig. 7(a), revealed that some Ge dots had elongated structure like the hut structure as indicated by arrows. A cross-sectional TEM image of such an elongated dot is shown in Fig. 7(b). The ratio between the diameter and the height of the Ge dot in this image is about $10: 1$, which is a typical value of the hut structure. Many of the dots, however, indicate an irregular shape; they don't have clear round shape of the domes, square bases of the pyramids or rectangular of the huts. Meanwhile, relatively large islands with about $60 \mathrm{~nm}$ in diameter, which are indicated by arrows in this image, are observed in Fig. 6(c). These islands are the dome structures. These results imply that the structure transition through post-annealing took place step-by-step and the Ge dot structure can be controlled by changing the thickness of the $\mathrm{C}$ top layers. The irregular structure observed in Fig. 6(b) is considered to be the intermediate structure between huts and domes, which are formed on the way of structure transition. This process is schematically illustrated in Fig. 8.

Here, it should be mentioned that small Ge dots with $10-20 \mathrm{~nm}$ in diameter were formed on the way of structure transition as shown in Fig. 6(b). Furthermore, the Ge dots were found to have high number density of $1 \times 10^{11} \mathrm{~cm}^{-2}$. Consequently, the Ge dots thus prepared have a 

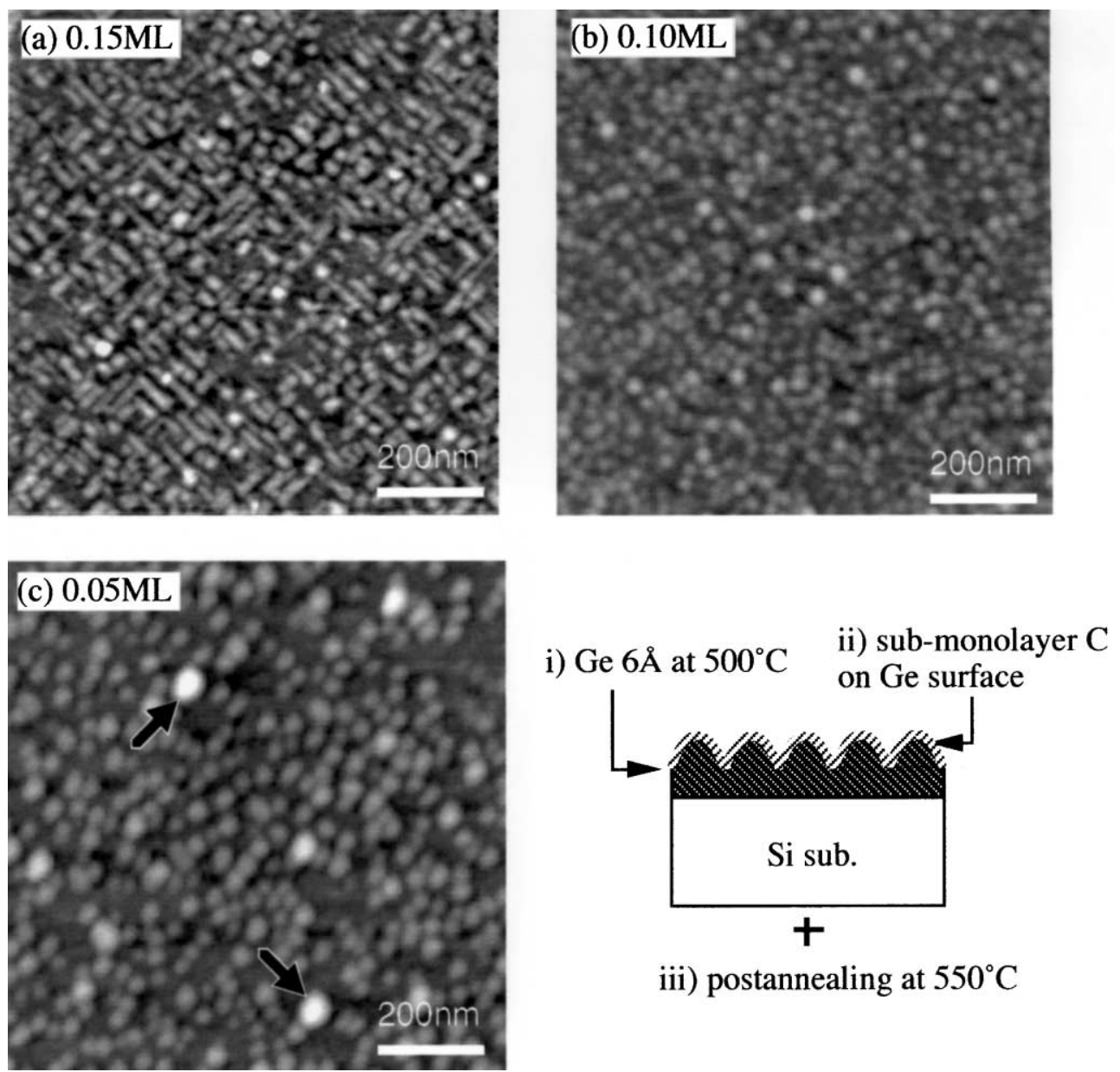

iii) postannealing at $550^{\circ} \mathrm{C}$

Fig. 6. AFM images of $\mathrm{Ge}$ dots with $\mathrm{C}$ top layers. $\mathrm{Ge}$ and $\mathrm{C}$ were deposited at $500^{\circ} \mathrm{C}$ and followed by post-annealing at $550^{\circ} \mathrm{C}$. The mean thickness of the $\mathrm{C}$ top layers are (a) $0.15 \mathrm{ML}$, (b) $0.10 \mathrm{ML}$ and (c) $0.05 \mathrm{ML}$. The sample setup is also illustrated. Elongated structure with the [ $\left[\begin{array}{ll}0 & 1\end{array}\right]$ direction was observed in Fig. 6(a), while the Ge dots tend to form round structure with decreasing $\mathrm{C}$ layer thickness. Some Ge dots showed dome structure as indicated by arrows in Fig. 6(c). These results indicate that the C top layer suppress the structure transition upon thermal annealing.

possibility for the quantum dots, whereas the size uniformity can stand further improvement.

It is worth emphasizing that the sub-monolayers $\mathrm{C}$ indicate quite distinct effects on the Ge dot structures. Namely, the C layers on the Ge wetting layer enhance the structure transition leading to dome formation even at low temperature. By contrast, those on the Ge surface suppress the structure transition upon thermal annealing. Latter effect is also considered to be relevant to the configuration energy of Ge dots. We deduced a possible model as follows. Strain fields on the Ge surface are inhomogeneous; the lattice strains on the top of the huts are relaxed and those around the edges of huts are compressive [22,23]. Therefore, the $\mathrm{C}$ atoms deposited on the Ge surface tend to agglomerate around the edges to accommodate the lattice strains. The elastic energy is compensated in this way particularly around the edges. The dot structure is stabilized upon thermal annealing and, as a result, the rearrangement of the Ge dot structure was suppressed. An extent of the strain compensation is dependent on the amount of the $\mathrm{C}$ atoms and can be precisely 

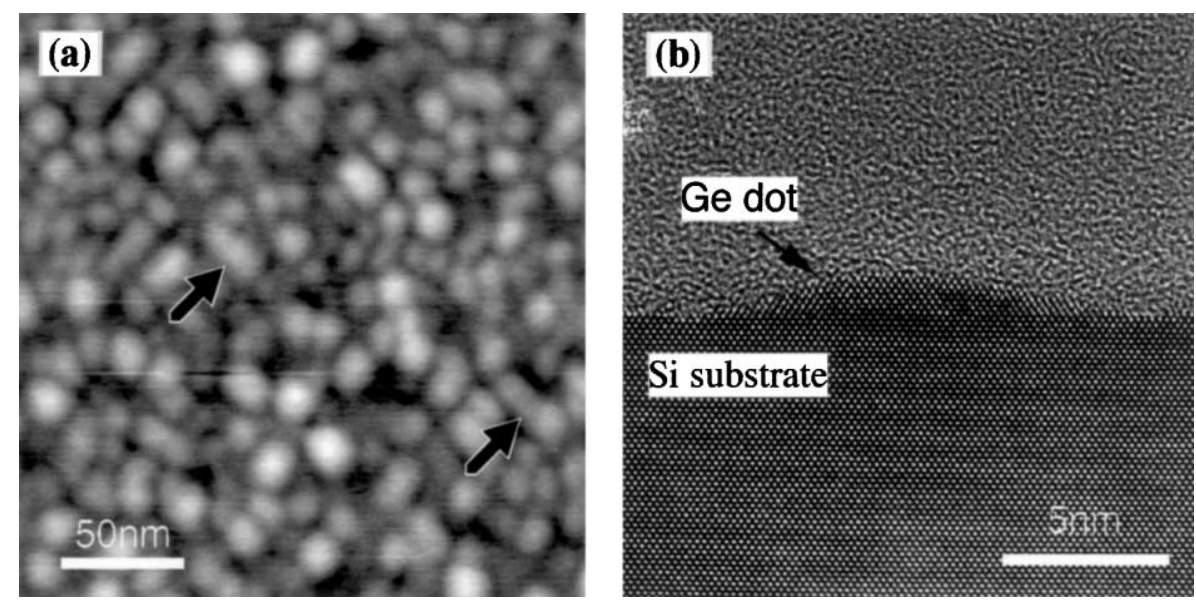

Fig. 7. Enlarged AFM image of Fig. 6(b). (a) Some Ge dots showed elongated structure which were regarded as the hut structure. (b) Cross-sectional TEM image of an elongated dot. The aspect ratio between the diameter and the height is about $10: 1$, which is the typical value of the hut structure.

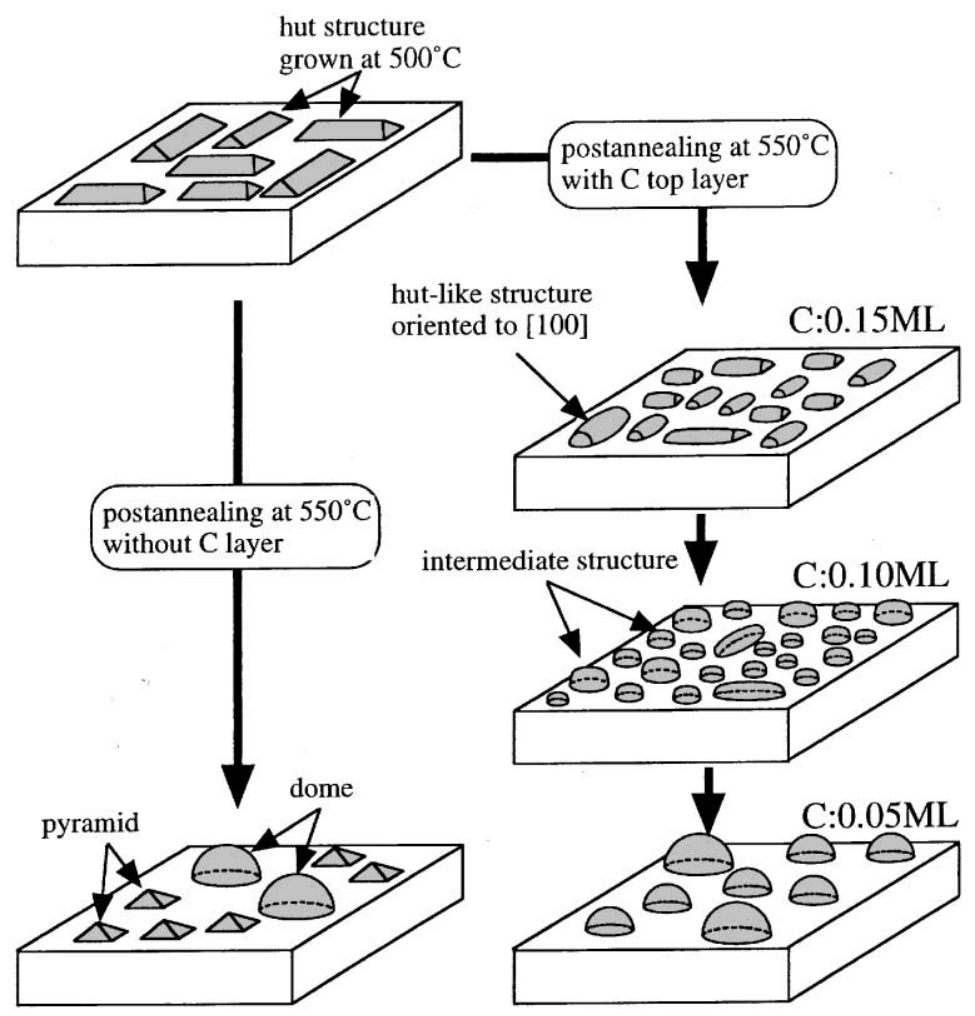

Fig. 8. Schematic illustration of structure transition upon post-annealing. The hut structure of pure $\mathrm{Ge}$ formed at $500^{\circ} \mathrm{C}$ rapidly transits to pyramids and domes upon $550^{\circ} \mathrm{C}$ post-annealing. The $\mathrm{C}$ top layers suppress the structure transition and enable a gradual control of the Ge dot structure. On the way of the structure transition, intermediate structure between huts and domes are observed. 

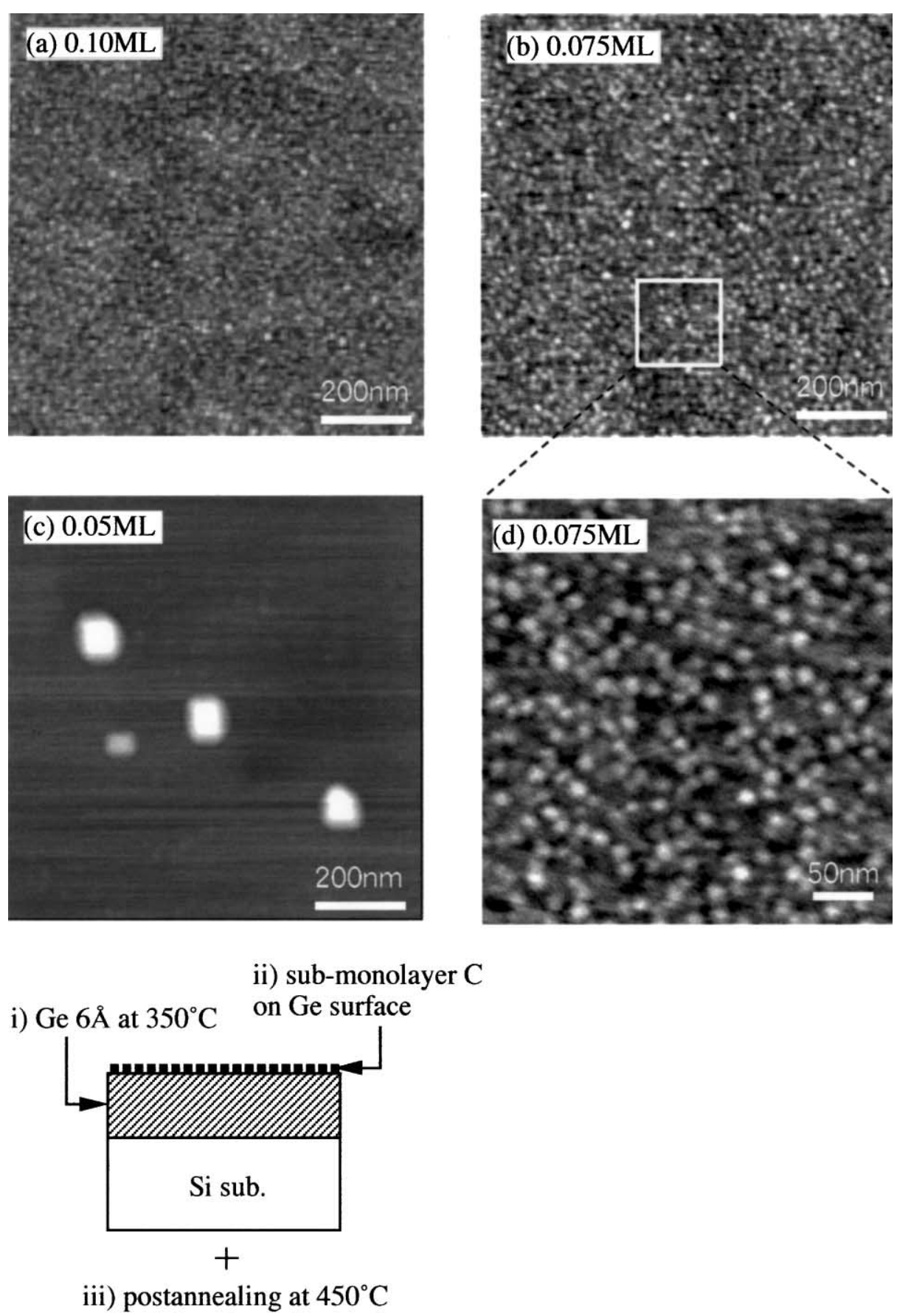

Fig. 9. AFM images of $\mathrm{Ge}$ dots with $\mathrm{C}$ top layers deposited at $350^{\circ} \mathrm{C}$ followed by post-annealing at $450^{\circ} \mathrm{C}$. The mean thickness of $\mathrm{C}$ top layers are (a) $0.10 \mathrm{ML}$, (b) $0.075 \mathrm{ML}$ and (c) $0.05 \mathrm{ML}$. The $\mathrm{C}$ top layer with $0.10 \mathrm{ML}$ prevented structure transition and twodimensional structure remained. The Ge layers tend to form 3D structures with decreasing C layer thickness as can be seen in Figs. 6(b) and (c). (d) Enlarged image of Fig. 9(b). $10 \mathrm{~nm}$-sized Ge dots with high density and high size uniformity were formed. 
modified by changing thickness of the $\mathrm{C}$ top layers. Thus, the $\mathrm{C}$ layers on the $\mathrm{Ge}$ surface made it possible the gradual structure transition. The irregular structure observed in Fig. 7(a) can be ascribed to the intermediate structure between huts and domes, which are hardly observed in the pure Ge films because the structure transition occurs abruptly.

On the basis of these experimental results, we combined two effects; the $\mathrm{C}$ layer on the $\mathrm{Ge}$ surface and low-temperature deposition with postannealing. Fig. 9 shows the AFM images of another type of samples-III. For these samples, both of the Ge layers and the $\mathrm{C}$ top layers were deposited at $350^{\circ} \mathrm{C}$. The mean thickness of the $\mathrm{C}$ top layers were (a) $0.10 \mathrm{ML}$, (b) $0.075 \mathrm{ML}$ and (c) $0.05 \mathrm{ML}$, respectively. Post-annealing was carried out at $450^{\circ} \mathrm{C}$ immediately after deposition. These images show the similar tendency of Fig. 6, i.e., the C top layers prevent the structure transition upon thermal annealing. For the case of pure Ge films deposited at $350^{\circ} \mathrm{C}$, the structure transition was drastically occurred from the $2 \mathrm{D}$ film to the huge islands as shown in Fig. 2. However, only 0.10 ML of $\mathrm{C}$ layer on the Ge surface resulted in the high stability against post-annealing. As shown in Fig. 9(a), the Ge layer remained two-dimensional even after $450^{\circ} \mathrm{C}$ post-annealing, while the fine roughness was observed. On the other hand, $0.05 \mathrm{ML}$ of $\mathrm{C}$ was insufficient to suppress the structure transition. In Fig. 9(c), the large Ge islands were observed in a similar manner of the pure Ge shown in Fig. 2(b).

In Fig. 9(b), small Ge dots were formed as an intermediate between the 2D films and the large 3D islands. The Ge dots thus prepared have the highest number density of $2 \times 10^{11} \mathrm{~cm}^{-2}$ among the samples described here. An enlarged image is shown in Fig. 9(d). The diameter of the Ge dots thus formed is about $10 \mathrm{~nm}$ on average. Moreover, the size uniformity was obviously improved compared with that of Fig. 7(a). These results suggest that a three-step procedure: (i) low temperature Ge deposition, (ii) $\mathrm{C}$ top layer and (iii) post-annealing is a promising technique for $10 \mathrm{~nm}$-sized quantum dot formation.

The effect of the $\mathrm{C}$ top layers for the low temperature deposited $\mathrm{Ge}$ is probably different from that discussed above. The elastic energy is released by introducing some defects in the as-deposited Ge layers. This is the reason for the $\mathrm{Ge}$ layers to have two-dimensional structure. Therefore, the $\mathrm{C}$ atoms are considered to distribute homogeneously on the Ge surface. In the meantime, 3D islanding are induced via postannealing. Then, the $\mathrm{C}$ atoms may act as nucleation sites for the Ge dot formation. Another possibility is as follows. The strain fields are induced around the Ge dots as the structure is rearranged to $3 \mathrm{D}$ during the post-annealing. Then, the $\mathrm{C}$ atoms redistribute around the Ge dots to compensate the lattice strains and prevent abrupt structure modification. Further experiments, in particular for $\mathrm{C}$ atoms distribution on $\mathrm{Ge}$, are required to understand the $\mathrm{C}$ effect on the Ge dot structure in more detail.

\section{Summary}

We investigated the Ge dots formation process through multistep procedure, which were lowtemperature deposition of Ge, post-annealing, sub-monolayer $\mathrm{C}$ deposition on the Ge wetting layer and on the Ge top layer surface. The $\mathrm{C}$ layers on the Ge wetting layers were found to enhance the Ge dot structure transition from hut to dome. As a result, small Ge domes were formed even below $500^{\circ} \mathrm{C}$. On the contrary, the $\mathrm{C}$ layers on the Ge surface were effective to suppress the structure transition of the Ge dots upon post-annealing. The structure of Ge layers deposited at low temperature was readily modified by post-annealing. By combining these phenomena and optimizing experimental conditions, including Ge deposition temperature, the thickness of the $\mathrm{C}$ layers and post-annealing temperature, the $10 \mathrm{~nm}$-sized Ge dots growth with high density of $2 \times 10^{11} \mathrm{~cm}^{-2}$ and with high size uniformity was achieved on the $\mathrm{Si}$ substrate. In the conventional self-assemble process, gas-phase atoms are supplied on the substrate directly to form solid-state islands. High mobility of gas-phase atoms makes it difficult to control the dot size and density. On the other hand, the multistep procedure described here utilized a kind of solid-state reaction, which open another 
approach to control more precisely the size and structure of nanoscale crystalline dots.

\section{References}

[1] G. Abstreiter, P. Schittenhelm, C. Engel, E. Silveira, A. Zrenner, D. Meertens, W. Jäger, Semicond. Sci. Technol. 11 (1996) 1521.

[2] M. Hammar, F.K. LeGoues, J. Tersoff, M.C. Reuter, R.M. Tromp, Surf. Sci. 349 (1996) 129.

[3] A.I. Yakimov, A.V. Dvurechenskii, A.I. Nikiforov, O.P. Pchelyakov, Thin Solid Films 336 (1998) 332.

[4] T.I. Kamins, E.C. Carr, R.S. Williams, S.J. Rosner, J. Appl. Phys. 81 (1997) 211

[5] M. Goryll, L. Vescan, K. Schmidt, S. Mesters, H. Lüh, Appl. Phys. Lett. 71 (1997) 410.

[6] G. Medeiros-Ribeiro, A.M. Bratkovski, T.I. Kamins, D.A.A. Ohlberg, R.S. Williams, Science 279 (1998) 353.

[7] F.M. Ross, J. Tersoff, R.M. Tromp, Phys. Rev. Lett. 80 (1998) 984.

[8] X. Wang, Z. Jiang, H. Zhu, F. Lu, D. Huang, X. Liu, C. Hu, Y. Chen, Z. Zhu, T. Yao, Appl. Phys. Lett. 71 (1997) 3543 .
[9] O.G. Schmidt, C. Lange, K. Eberl, O. Kienzle, F. Ernst, Appl. Phys. Lett. 71 (1997) 2340.

[10] O.G. Schmidt, S. Schieker, K. Eberl, O. Kienzle, F. Ernst, Appl. Phys. Lett. 73 (1998) 659.

[11] O.G. Schmidt, K. Eberl, Appl. Phys. Lett. 73 (1998) 2790.

[12] O. Leifeld, E. Müller, D. Grützmacher, B. Müller, K. Kern, Appl. Phys. Lett. 74 (1999) 994.

[13] O. Leifeld, R. Hartmann, E. Müller, E. Kaxiras, K. Kern, D. Grützmacher, Nanotechnology 10 (1999) 122.

[14] J. Drucker, S. Chaparro, Appl. Phys. Lett. 71 (1997) 614.

[15] G. Capellini, L. Di Gaspare, F. Evangelisti, E. Palange, Appl. Phys. Lett. 70 (1997) 493.

[16] M. Tomitori, K. Watanabe, M. Kobayashi, O. Nishikawa, Appl. Surf. Sci. 76/77 (1994) 322.

[17] D.E. Jesson, M. Kästner, B. Voigtländer, Phys. Rev. Lett. 84 (2000) 330.

[18] V.A. Markov, O.P. Pchelyakov, L.V. Sokolov, S.I. Stenin, S. Stoyanov, Surf. Sci. 250 (1991) 229.

[19] F.K. LeGoues, J. Tersoff, M.C. Reuter, M. Hammar, R. Tromp, Appl. Phys. Lett. 67 (1995) 2317.

[20] Y. Wakayama, G. Gerth, P. Werner, U. Gösele, L.V. Sokolov, Appl. Phys. Lett. 77 (2000) 2328.

[21] R. Butz, H. Lüth, Thin Solid Films 336 (1998) 69.

[22] Y. Chen, J. Washburn, Phys. Rev. Lett. 77 (1996) 4046.

[23] A.G. Cullis, Mat. Res. Soc. Bulletin 21 (1996) 21. 